\section{Längere Rezidivfreiheit beim lokal begrenzten Hochrisiko-Prostatakarzinom}

\author{
Frühe risikoadaptierte chemotherapeutische Strategien galten bisher \\ nicht als Standard beim lokalisierten Hochrisiko-Prostatakarzinom. Erst- \\ mals wurde denr Effekt der Kombination aus Androgendeprivation, \\ Docetaxel und Estramustin auf das rezidivfreie Überleben untersucht.
}

A ndrogenentzug (ADT) plus Strahlentherapie erwies sich bei Patienten mit einem lokalisierten Hochrisiko-Prostatakarzinom (PCa) als der alleinigen ADT überlegen. Die Kombination von Docetaxel mit dem Zytostatikum Estramustin erhöht Studien zufolge das Gesamtüberleben von Patienten mit einem kastrationsresistenten PCa im Vergleich $\mathrm{zu}$ einer Therapie auf Mitoxantron-Basis.

Basierend auf diesen Daten untersuchte ein Team um Karim Fizazi im Rahmen einer randomisierten, kontrollierten Phase-III-Studie den Effekt der Addition von Docetaxel/Estramustin zur ADT auf das rezidivfreie Überleben von
Patienten mit einem lokal begrenzten Hochrisiko-PCa. Sie rekrutierten 413 unbehandelte Patienten mit mindestens einem Risikofaktor (Stadium T3-T4, Gleason-Score $\geq 8$, PSA-Spiegel $>20 \mathrm{ng} /$ $\mathrm{ml}$ oder pathologisch positive Lymphknoten). 207 Patienten erhielten eine ADT (10,8 mg Goserelin alle 3 Monate über 3 Jahre) und 4 Zyklen Docetaxel (70 $\mathrm{mg} / \mathrm{m}^{2}$ an Tag 2) plus Estramustin (täglich $10 \mathrm{mg} / \mathrm{kg}$ an den Tagen 1-5, q3w). Im Vergleichsarm $(n=206)$ wurde nur die ADT verabreicht.

Nach median 8,8 Jahren hatten 43\% der Patienten im Kombinationsarm und $54 \%$ in der ADT-Gruppe ein Ereignis er- litten (Rezidiv oder Tod). Das rezidivfreie Überleben nach 8 Jahren fiel in der Kombinationsgruppe mit $62 \%$ günstiger aus als in der Vergleichsgruppe (50\%). Insgesamt wurde das ADT/Docetaxel/Estramustin-Regime gut vertragen.

Fazit: Nach Kenntnisstand der Forscher zeigten ihre Daten erstmals eine signifikante Verlängerung des rezidivfreien Krankheitsverlaufs bei Patienten mit einem lokal begrenzten Hochrisiko-PCa durch Integration von Docetaxel und Estramustin in gängige Behandlungsregimes. Ob sich dieser Vorteil langfristig hemmend auf die Metastasierungstendenz und positiv auf das Gesamtüberleben auswirkt, kann nur in weiterführende Studien mit einem längeren Followup eruiert werden. Wolfgang Zimmermann

Fizazi K et al. Androgen deprivation therapy plus docetaxel and estramustine versus androgen deprivation therapy alone for high-risk localised prostate cancer (GETUG 12): a phase 3 randomised controlled trial. Lancet Oncol. 2015;16(7):787-94.

\title{
PCa: Ibandronat gut bei Knochenschmerzen
}

\section{Bei lokalisierten Knochenschmerzen aufgrund von Metastasen eines Prostatakarzinoms ist die Radiotherapie Standard. Nun wurde die Effektivität einer einzelnen Infusion des Bisphosphonats Ibandronat getestet.}

Konsting nochenmetastasen sind eine häufige und schwerwiegende Komplikation beim Prostatakarzinom (PCa). Dass sich metastasenbedingte Knochenschmerzen mit Bisphosphonaten gut kontrollieren lassen, konnte schon in früheren Studien gezeigt werden, ein direkter Vergleich zur Radiotherapie stand jedoch aus.

In einer Studie erhielten nun 470 Patienten mit metastasenbedingten Knochenschmerzen randomisiert entweder eine Einzeldosis Radiotherapie (8 Gy) oder eine einzelne Infusion von $6 \mathrm{mg}$ Ibandronat. Die Schmerzen wurden zu Beginn der Studie sowie nach 4, 8, 12, 26 und 52 Wochen mithilfe des BPI („,brief pain inventory“) erhoben. Wer innerhalb von 4 Wochen nicht ansprach, durfte die jeweils andere Therapie durchführen. Anhand der Kriterien der World Health Organization (WHO) (pain lad- der) und des EAS („effective analgesic score") wurde die Schmerzreduktion über den Konsum von Analgetika und einen Schmerzscore im Vergleich zur Baseline ermittelt. Maximal erlaubt war eine Differenz von $\pm 15 \%$ zwischen den Gruppen. Komplettes Ansprechen war als Schmerzscore von 0 mit stabilem oder reduziertem Analgetikaverbrauch definiert. Als partielles Ansprechen galt eine Reduktion im Schmerzscore von mindestens 2 Punkten mit stabilem oder reduziertem Analgetikaverbrauch oder - bei einer Schmerzreduktion von höchstens 1 Punkt - eine Reduktion des Analgetikaverbrauchs von mindestens $25 \%$.

Nach 4 und 12 Wochen gab es zwischen den Behandlungsgruppen keine statistisch signifikanten Unterschiede in der Schmerzreduktion - weder anhand der WHO-Kriterien noch unter Verwen- dung des EAS. Bei der Radiotherapie trat das Ansprechen schneller ein.

Hinsichtlich der Stärke von Nebenwirkungen gab es keine Unterschiede, aber in den Therapiearmen traten unterschiedliche Nebenwirkungen auf. Die Lebensqualität war nach 4 und 12 Wochen vergleichbar. Auch das Gesamtüberleben war in beiden Gruppen ähnlich. Es war bei Patienten, die wegen fehlenden Ansprechens nach 4 Wochen eine erneute Behandlung mit der jeweils anderen Therapie erhielten, besser als bei jenen, die auf eine weitere Behandlung verzichteten.

Fazit: Eine Einzelinfusion Ibandronat kann Knochenschmerzen infolge von Metastasen bei Prostatakarzinom ähnlich gut lindern wie eine einzelne Bestrahlung. Nach Ansicht der Forscher steht somit das Bisphosphonat als Option zur Verfügung, wenn eine Radiotherapie nicht möglich ist. Kathrin von Kieseritzky

Hoskin P et al. A Multicenter Randomized Trial of Ibandronate Compared With Single-Dose Radiotherapy for Localized Metastatic Bone Pain in Prostate Cancer. J Natl Cancer Inst. 2015; 107(10). pii: djv197. 